\title{
SOLVING A PERIODIC CAPACITATED VEHICLE ROUTING PROBLEM USING SIMULATED ANNEALING ALGORITHM FOR A MANUFACTURING COMPANY
}

Erdal Aydemir

erdalaydemir@sdu.edu.tr Suleyman Demirel University, Engineering Faculty, Dept. of Industrial Engineering, 32260, Isparta, Turkey.

\section{Kenan Karagul}

kkaragul@pau.edu.tr

Pamukkale University, Dept. of Logistics, Honaz, Denizli, Turkey.

\section{ABSTRACT}

Goal: This paper aims to implement a periodic capacitated vehicle routing problem with simulated annealing algorithm using a real-life industrial distribution problem and to recommend it to industry practitioners. The authors aimed to achieve high-performance solutions by coding a manually solved industrial problem and thus solving a real-life vehicle routing problem using Julia language and simulated annealing algorithm.

Design / Methodology / Approach: The vehicle routing problem (VRP) that is a widely studied combinatorial optimization and integer programming problem, aims to design optimal tours for a fleet of vehicles serving a given set of customers at different locations. The simulated annealing algorithm is used for periodic capacitated vehicle routing problem. Julia is a state-of-art scientific computation language. Therefore, a Julia programming language toolbox developed for logistic optimization is used.

Results: The results are compared to savings algorithms from Matlab in terms of solution quality and time. It is seen that the simulated annealing algorithm with Julia gives better solution quality in reasonable simulation time compared to the constructive savings algorithm. Limitations of the investigation: The data of the company is obtained from 12 periods with a history of four years. About the capacitated vehicle routing problem, the homogenous fleet with $\mathbf{3 0 0 0}$ meters/vehicle is used. Then, the simulated annealing design parameters are chosen rule-of-thumb. Therefore, better performance can be obtained by optimizing the simulated annealing parameters.

Practical implications: In this study, a furniture roving parts manufacturing company that have 30 customers in Denizli, an industrial city in the west part of Turkey, is investigated. Before the scheduling implementation with Julia, the company has no effective and efficient planning as they have been using spreadsheet programs for vehicle scheduling solutions. In this study, the solutions with Julia are used in practice for the distribution with higher utilization rate and minimum number of vehicles. The simulated annealing and savings algorithms are compared in terms of solution time and performance. The savings algorithm has produced better solution time, the simulated annealing approach has minimum total distance objective value, minimum number of required vehicles, and maximum vehicle utilization rate for the whole model. Thus, this paper can contribute to small scale business management in the sense of presenting a digitalization solution for the vehicle scheduling solution. Also, Julia application of simulated annealing for vehicle scheduling is demonstrated that can help both academicians and practitioners in organizations, mainly in logistics and distribution problems.

Originality / Value: The main contribution of this study is a new solution method to capacitated vehicle routing problems for a real-life industrial problem using the advantages of the high-level computing language Julia and a meta-heuristic algorithm, the simulated annealing method.

Keywords: Capacitated Vehicle Routing Problem; Simulated Annealing Algorithm; Julia Programming Language. 


\section{INTRODUCTION}

Nowadays, once starting the rapidly spreading implementations of Industry 4.0 for the companies, the supply chain management 4.0 procedure is also developed for which a bibliometric analysis reinforce the growing importance of the topic. According to Frazzon et al. (2019), the approaches of big data, cloud computing, and Internet of Things are the most widely used technologies for the smart applications and digitalization of the supply chain process. At this phase, the routing solutions are also important for transportation management system by linking enterprise resource planning and warehouse management system.

The vehicle routing problem (VRP) is a combinatorial optimization and integer programming problem and belongs to NP-hard computational complexity class. Formerly, It was described and solved by Dantzig and Ramser (1959) as a linear programming problem. Five years after these novel description, Clarke and Wright (1964) proposed the Clarke and Wright Savings Algorithm that depends on the idea of computing the savings for linking two customers at the same route (Pichpibul and Kawtummachai, 2013). As generated from Travelling Salesman Problem with set of constraints, VRP aims to obtain the minimization of delivery costs of vehicles to customers at different locations from a depot. In the literature, VRP variants with various constraints, goals and states have been presented and solved. Thus, in the operational research area, VRP has numerous applications.

Both exact and heuristics based solution methods have been proposed to solve VRPs. In VRP solutions, vehicle capacity and/or route distance constraints are taken into consideration. For instance, in Capacitated Vehicle Routing Problem (CVRP) the aim is to provide the delivery process with known demands by optimizing the cost bearing in mind the vehicle capacity constraints (Cordeau et al., 2004). As in the case of VRP, exact and heuristic based solutions have been also used from the time when it is first defined for solving CVRP. The non-exact heuristics solution methods improve the computation time algorithm complexity with respect to exact solutions and add liability to vehicle capacity and route distance constraints (Barhant and Laporte, 2006; Laporte, 2009).

The VRP variants are classified and mainly demonstrated in Figure 1 where it is seen that various VRP variations have been proposed in the literature. As seen in Figure 1, the main VRP variants are the CVRP (Laporte et al., 1986; Mazzeo and Loiseau, 2004; Braysy and Gendreau, 2005; Şahin and Eroğlu, 2014; Karagul et al., 2016; Gayialis et al., 2019), multi-depot VRP (MDVRP) (Laporte et al., 1988; Wu et al., 2002; Silva Júnior et al., 2011; Allahyari et al., 2015; Lalla-Ruiz and Voß, 2019), split delivery VRP
(SDVRP) (Archetti et al., 2011; Bianchessi and Irnich, 2019), periodic VRP (PVRP) (Cacchiani et al., 2014; Rahimi-Vahed et al., 2015; Archetti et al., 2017), stochastic VRP (SVRP) with demands (VRPSD), time (VRPST) and customers (VRPSC) (Laporte et al., 1992; Bertsimas et al., 1995; Gendreau et al., 2016; Saint-Guillain et al., 2017). The variants of CVRP are time windows (VRPTW) (Dror, 1994; Gambardella et al., 1999; Alvarenga et al., 2007; Chen et al., 2017), distance-constrained VRP (DCVRP) (Kara and Derya, 2011; Bernal et al., 2018), VRP with pickup and delivery (VRPPD) (Ganesh and Narendran, 2007; Mosheiova, 1998; Katoh and Yano, 2006; Yanik and Bozkaya, 2014; Li et al., 2017) and VRP with backhauls (VRPB) (Toth and Vigo, 2002; Gribkovskaia et al., 2008; Reil et al., 2018). Furthermore, there are some variants such as VRP with heterogenous fleet size (Taillard, 1993; Salhi et al., 2014; Coelho et al., 2016; Penna et al., 2019), open VRP (Tarantilis et al., 2004; Letchford et al., 2007; Tyasnurita et al., 2017), rich VRP (Hartl et al., 2006; Koç et al., 2018; Sim et al., 2019), fuzzy VRP (Brito et al., 2009; Cao and Lai, 2010; Kuo et al., 2016), and swap-body VRP (Huber and Geiger, 2017; Absi et al., 2017). Nowadays, VRP with green transportation strategy has been attractively studied with minimizing the carbon emissions on routing (Erdogan and Miller-Hooks, 2012; Bektas et al., 2016; Ene et al., 2016; Koç and Karaoglan, 2016; Montoya et al., 2016; Karagul et al., 2019; Koç et al., 2019).

In this paper, a CVRP is solved using Julia programming language with simulated annealing algorithm under reasonable time and reasonable number of vehicles. As an instance, a furniture roving part manufacturing company has examined with 30 -customers for 12 periods with a homogenous fleet size from a depot. The rest of the paper is organized as follows. Section 2 introduces a mathematical model for the CVRP. Section 3 defines simulated annealing algorithms and Julia programming language solution approach, and Section 4 defines the proposed model and presents the simulations and analysis, followed by conclusions and further research in the last section.

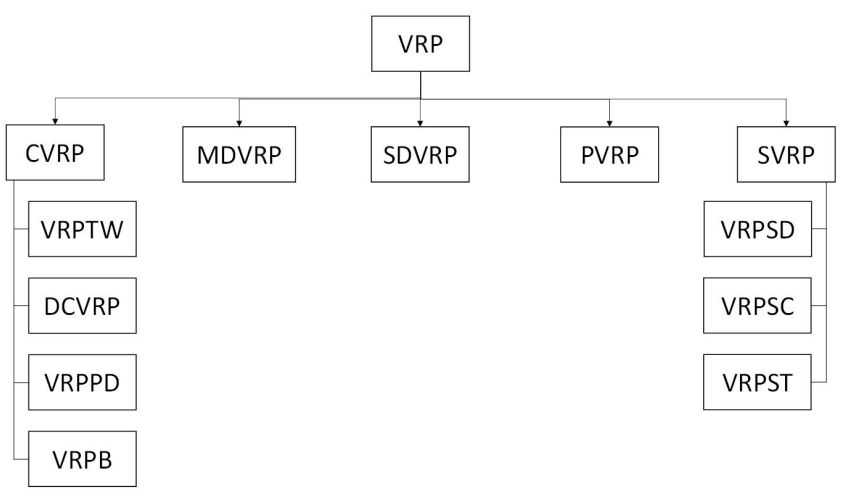

Figure 1. The VRP variants 


\section{CAPACITATED VEHICLE ROUTING PROBLEM}

Capacitated vehicle routing problem, CVRP, can be structured to start using undirected graph as $G=(V, E)$ where $V=\left\{v_{0}, v_{l}, v_{2}, v_{3}, \ldots, v_{n}\right\}$ and $E=\{(i, j): i, j \in V\}$ is a set of vertices. Here, $V=v_{0}$ is the depot with no demand level. When the cost for moving from $i$ to $j$ does not depend on the direction, undirected graph $G=(V, E)$ has an edge set $E=\{(i, j): i, j \in V, i<j\}$.

In CVRP, the total cost of the route of the vehicle is minimized under the following constraints:

- Each city on the route is visited by only one vehicle,

- Each route starts at the depot and ends at the depot,

- The total number of cities on a route, vehicle capacity, time windows and total service time are limited.

The CVRP mathematical model of the problem under these constraints is shown below (Laporte, 1992; Laporte and Semet, 2002; Toth and Vigo, 2002; El Hassani et al., 2008).

$$
\begin{array}{lc}
\min _{k \in K i \in V} \sum_{j \in V} d_{i j} x_{i, j}^{k} & \\
\sum_{k \in K} \sum_{j \in N} x_{i, j}^{k}=1 & \forall i \in V \\
\sum_{j \in V} x_{i, j}^{k}+\sum_{j \in V} x_{j, i}^{k}=1 & \forall i \in V, k \in K \\
\sum_{k=1}^{K} \sum_{j=1}^{N} x_{0, j}^{k}=K & \\
\sum_{j \in V} x_{0, j}^{k}=1 & \forall k \in K \\
\sum_{j \in V} x_{j, n+1}^{k}=1 & \forall k \in K \\
x_{i, j}^{k}=1=> & y_{i}-q_{j}=y_{j}, \\
y_{0}=Q, 0 \leq y_{i} & \forall i, j \in V, \forall k \in K \\
\sum_{i=1}^{N} q_{i} \sum_{j=0, j \neq i}^{N} x_{i, j}^{k} \leq Q & \forall i \in V \\
x_{i, j}^{k} \in\{0,1\} & \forall \in\{1, \ldots, m\} \\
\end{array}
$$

where

$$
x_{i, j}^{k}=\left\{\begin{array}{lc}
1, & \text { if the vehicle } k \text { visits from customer } i \text { to customer } j \\
0, & \text { otherwise }
\end{array}\right.
$$

$y_{i}^{k}=\left\{\begin{array}{lc}1, & \text { if the vehicle } k \text { services to customer } i \text { demand } \\ 0, & \text { otherwise }\end{array}\right.$

and $d_{i j}$ is the distance between customers $i$ to $j$. The $i$-th customer has non-negative demand quantity $q_{i}$ in CVRP. The fleet size $K=\left\{k_{1}, k_{2}, \ldots, k_{m}\right\}$ is assumed to be homogenous that means all $m$ vehicles have the same capacity, $\forall k_{i} \in K$.

The VRP is given in (1)-(10) as a constraint optimization problem. (1) is used for the minimization of the route cost in which the delivery vehicles are traveling. The constraint in (2) is used for each vehicle to serve an $i-j$ connection, (3) is used to avoid returning to the same node. The constraint (4) guarantee that the number of the vehicles arriving at every customer and entering the depot is equal to the number of the vehicles leaving. The constraints (5) and (6) guarantee that each vehicle can leave the depot and visit the j-th node only once. The constraint (7) shows the remaining capacity after the vehicle came $i$-th node from $j$-th node when the vehicle is assigned to the $i-j$ connection. The constraint (8) guarantees that the initial capacity of the vehicles is $Q$. According to the constraint (9), the total demand of customers assigned to a vehicle will not exceed the capacity of the vehicle. The constraint (10) is an integer constraint for the variable $x_{i, j}^{k}$.

Many methods have been developed for the solution of CVRP. The methods that provide the optimal solution are categorized as the exact solution methods. On the other hand, the methods that give the near-optimal results are classified as the heuristic solution method. From a literature review, it is seen that branch \& cut and branch \& bound algorithms and dynamic programming models and set partition algorithms are frequently used as the exact solution methods. Although it is also used for the final solution, the preferred savings as a solution provider are the closest neighbor, two-stage method, and the petal intuitive classical intuition. In addition, in the third and the last group, metaheuristics such as genetic algorithms, simulated annealing, tabu search, particle swarm optimization such as ant colony and artificial bee colony, local search and acceptance threshold are also other methods used to solve CVRP. Metaheuristics methods are algorithms generally inspired by natural events to solve complex optimization problems that cannot be solved in a reasonable time by precise mathematical solution methods. Especially in large and integrated structures, the metaheuristics methods are the most practical way to solve real-life problems. The aim of these methods is to investigate the solution space effectively and to provide solutions that are close to the optimal. Being easy to understand and practical, it is widely used today because it can be used with slight changes in the solution of different problem types. The metaheuristic methods 
can be classified in terms of the inspiration used (natural or artificial), the initial solution used (population or single solution), the objective function used (dynamic, static), the neighborhood structure (single, multiple) and memory status (memory, memory-free) (Blum and Roli, 2003). In the last three decades, genetic algorithms (Holland, 1975), simulated annealing (Kirkpatrick et al., 1983), tabu search (Glover and McMillan, 1986), artificial immune system (Farmer et al., 1986), ant colony (Dorigo et al., 1991), particle swarm (Kennedy and Eberhart, 1995), artificial bee colony (Karaboga, 2005) are the frequently used metaheuristic methods in the solution of integrated optimization problems.

In this paper, simulated annealing algorithm is applied for solving the CVRP problem with a real-life industrial model and its results are obtained with minimizing the sum of the total distance.

\section{SOLUTION APPROACHES}

\subsection{Simulated Annealing (SA) Algorithm}

In general, analytical methods don't provide the optimal solution for the CVRP at a reasonable time. Thus, heuristics and metaheuristic approaches are used for solving combinatorial optimization problems. The goal of heuristics/metaheuristics approach is to carry out the solution space and to provide convenient results close to the optimal solution well planned.

In this study, simulated annealing (SA) algorithm which was first applied by Kirkpatrick et al. (1983) is used that is efficient metaheuristic approach that can be applied to solve CVRP. The method, which is named after metallurgical science and the annealing of metals, is often used for discrete optimization problems. SA is based on the physical annealing treatment which is using widely known metropolis algorithm in inner-loop of the algorithm (Johnson et al., 1989). SA has generally used to solve combinatorial optimization problems the same as other heuristics/metaheuristics approaches. Fundamental stone of SA algorithm is the dynamically decreasing iterations of the $p$ value, which is likely to accept the bad solution instead of sacrificing the good solution. When this arrangement is made, in the first parts of the problem solution there is a lot of leap between the solution regions, and when the number of iterations increases and the solutions we get are at a very good level, it approaches 0 and so our search zone becomes narrower. The basic principle of the annealing algorithm is precisely this. Annealing process based on the solidifying which is make liquefied by high and efficient heating treatment. Material change with the high temperature that mean internal particles and shape imbalances. Next phase of the process, it is systematically decreased temperature by system until the steady state condition. So, the possibility of selecting a bad solution is systematically reduced by temperature. Temperature is an expression due to iteration (usually smooth or logarithmic decreasing). Similarly, atoms is getting balance which is steady state condition in each iteration temperature. Finally, internal energy of the material is down minimum point because of the system heat's catch the environmental temperature(Lin and Fei, 2012; Şahin and Eroğlu, 2014; Karagul et al., 2019).

Osman (1993) developed a hybrid simulated annealing algorithm with the tabu search algorithm for solving a CVRP. Zeng et al. (2005) proposed an assignment-based local search algorithm used with simulated annealing. Tavakkoli-Moghaddam et al. (2007) developed a mixed integer linear model for CVRP, where a customer's demands can be divided into more than one vehicle and searched a possible solution with simulated annealing. Leung et al. (2010) developed a simulated annealing-based solution that considers the two-dimensional loading constraints for the integrated solution of vehicle loading and routing problems, which are two major problems of distribution logistics. Then, Tavakkoli-Moghaddam et al. (2011) presented a novel mathematical model for a competitive VRPTW to optimize routes considering the minimum travel cost and maximum sale using simulated annealing (SA) algorithm. By using both a small and a large dataset, it is shown that the SA algorithm can find useful solutions for VRPTW in a shorter time than exact methods. A multi-objective variant of the VRPTW based on the simulated annealing, namely, the multiple temperature Pareto simulated annealing is proposed and solved on the Solomon's datasets by Baños et al. (2013) where the travelled cost and the imbalance in the distances travelled by the vehicles, and in the loads delivered by them is optimized at the same time. Then, Vincent et al. (2017) developed simulated annealing with a restart strategy to solve this problem for hybrid VRP that is an extension of the green VRP. Their strategy determines the acceptance probability of a worse solution using the Boltzmann function at first and, employs the Cauchy function to determine the acceptance probability of a worse solution at the second part of strategy. Their study shows that the proposed SA approach has effectively solved the problem on vehicle type and the number of electric charging stations that have an impact on the total travel cost. The initial temperature, the number of solutions to be produced at each temperature, the temperature reduction function, and the stop criterion are the four parameters of SA.

The convergence of SA is given as in Figure 2. Graph showed to readers, convergence realized step by step in each iteration. The SA based solution algorithm for CVRP is given as follows in Chart 1 which includes pseudo-code and 


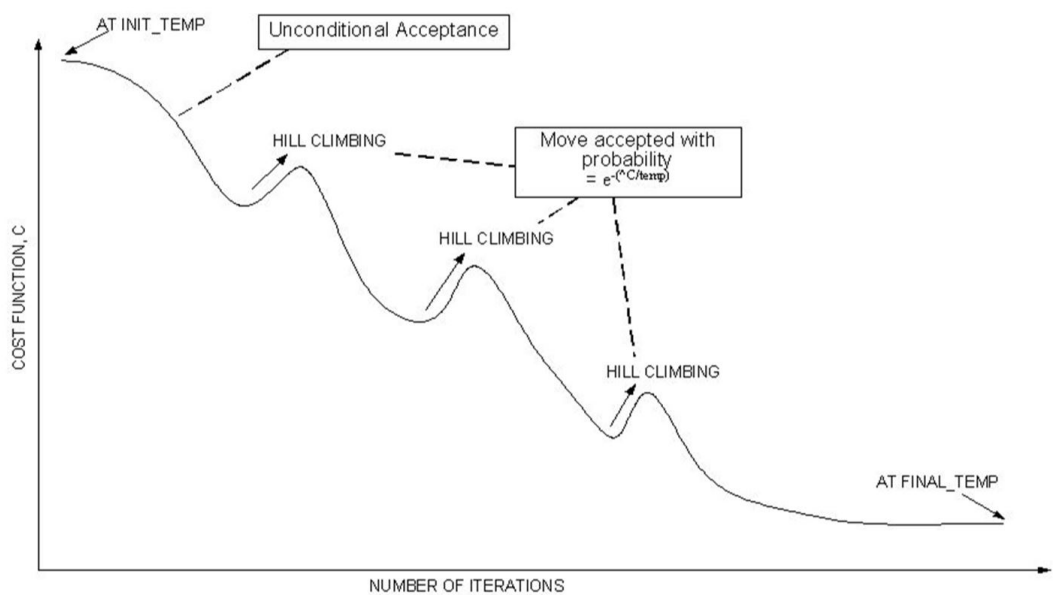

Figure 2. Convergence of simulated annealing algorithm (Abdulal et al., 2012)

the values of the simulation parameters in detail. The SA method has four parameters: the initial temperature, the number of solutions to be produced at each temperature, the temperature decrease function, and the halt condition. The convergence of SA is given as in Figure 2 and the SA based solution algorithm for CVRP is given as follows in Chart 1 which includes Matlab/Octave code like as pseudo-code and trivial optimized running parameters. The code is easy readable and understable.

\subsection{Julia Programming Language}

Modern programming languages have worked with stylish design objects and their compiler tools have reasonable performance. However, to provide a working environment for different disciplines or even for interdisciplinary studies, high performance scientific computing tools should present a user-friendly coding environment and syntax for research studies. In computer programming, a scientific language is a programming language especially optimized for mathematical contents such as linear algebra, dynamic equations, differential equation solvers and matrices used in scientific computing. Julia programming language which is carried out at the Julia Lab at Massachusetts Institute of Technology (MIT) in 2012, is a high-level, high-performance dynamic language suitable for scientific computation. In Julia, there are unexpectedly many ways in which utterings in the language can be used that makes Julia a flexible language. Being a free and MIT licensed open source software is another advantage of Julia. The custom software developed with Julia programming language can be compiled to a native code for multiple platforms via a low level virtual machine. In Julia, the user-defined types are as fast and compact as builtins. In Julia, the vectorization of the code is not necessary for computation performance; the de-vectorization of the code is also fast. Julia is designed for parallelism and distributed
Chart 1. Pseudo-code for Simulated Annealing for the CVRP $\mathbf{m V R P}=$ readVRP(TestProblemFile)

$\%$ mVRP.xy: VRP custormers and depot coordinates

$\%$ mVRP.q: Customers demand quantities

$\%$ mVRP.Q: Vehicle capacity

$\%$ mVRP.I: number of customers

$\%$ mVRP.J: number of vehicle

$\mathbf{m}=\mathrm{I}+\mathrm{J}-1$ : candidate solution size

Generate an initial solution $\mathrm{X}_{0}=$ shuffle[1:m]

Select an initial temperature and reduction formula $\alpha$

Repeat

Repeat

Create a neighbour $\mathrm{X}$ by randomly changing the caster

adjustment of a random heat

Evaluate neighbour using the heuristic discussed previously

If neighbour is an improvement over $X_{0}$

$\mathrm{X}_{0}=\mathrm{X}$

Else

Generate random number from Uniform Distribution $(0,1)$

If $R<\exp \left(\left(f(X)-f\left(X_{0}\right)\right) / t\right)$ then

$\mathrm{X}_{0}=\mathrm{X}$

Until iteration count $=$ nrep

Set $t=\alpha(t)$

Until stop condition=true

computation. Also, the $C$ functions can be directly called without wrappers or special APIs.

For a performance comparison of Julia and typical dynamic programming languages, the results of micro-benchmarks such as sorting, string parsing, numerical loops, random number generation, recursion, array operations, and 


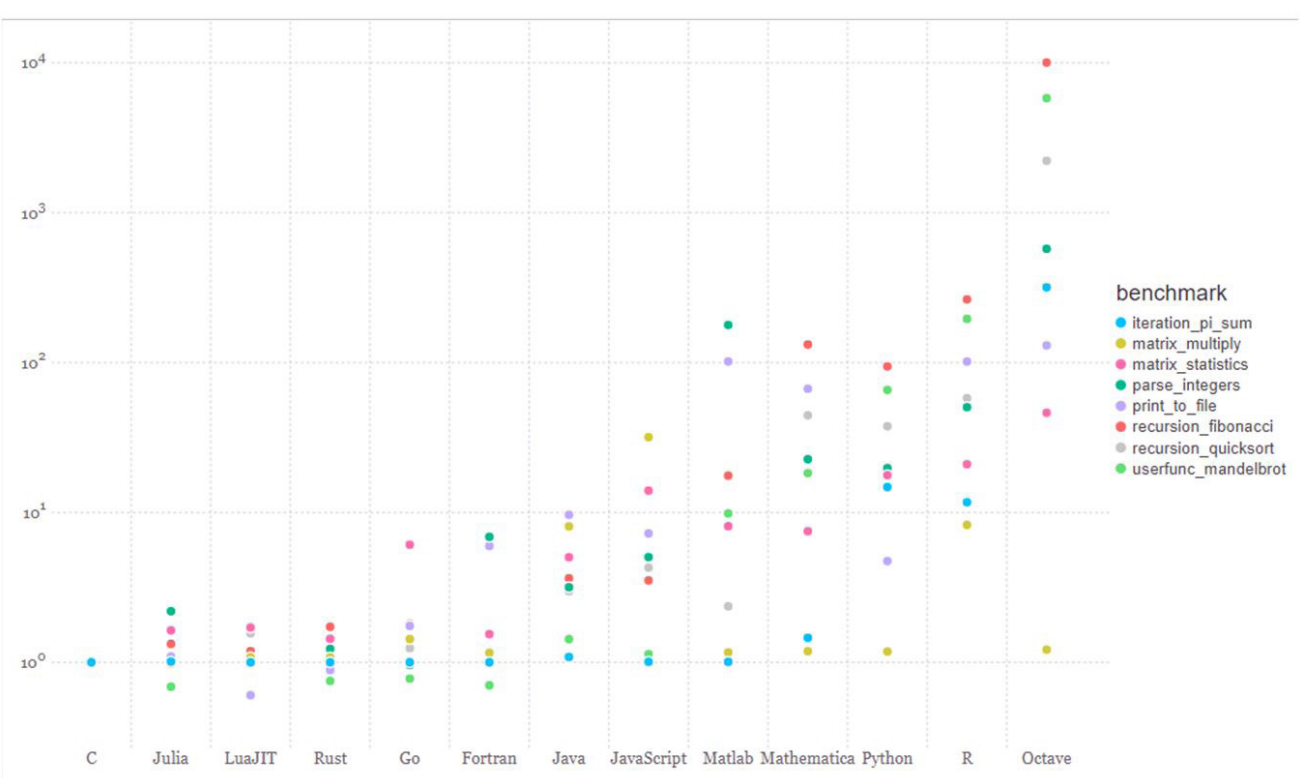

Figure 3. The benchmark results of Julia and typical dynamic languages (Julia Language, 2018).

function calls from the Julia homepage are given in Figure 3 (Julialang.org). These micro-benchmarks have related to do test compiler performance on a range of common code patterns, (Julialang.org).

Julia's ease of use and computation time advantages over other languages mentioned above makes it a good candidate for general purpose programming in addition to scientific analysis. From security point of view, there have been a few interesting issues that have come up that indicate that a deeper exploration of Julia in a potentially untrusted, multi-user, multi-threading environments such as Web applications, mobile platforms is warranted. However, the logistics optimization toolbox used for solving the CVRP is limited only for scientific usage for a standalone, single-user environment. Therefore, these security issues are not considered in this study.

Consequently, it is seen that Julia has more effective working fields such as machine learning, data analysis, parallel computing and scientific domains besides using general purposes and visualization. So, in this paper, Julia language is preferred to start with developing a new logistics toolbox and this paper is also presented for this purpose.

\section{NUMERICAL RESULTS}

As a case study, a furniture roving part manufacturing company which is located in Denizli, Turkey is considered.
The company has the demand values from 30-customers for 12 periods as seen in Table 1 . A sample photo of the furniture roving part is given as in Figure 4, the total demand fluctuations are given for each period as in Figure 5 where the seasonal and economic fluctuations can be observed. As seen in Figure 5, there are 12 periods corresponding to four years. Thus, each period belongs to four months. Appendix A shows the distances from depot to customers and also between each other.

The programs run on Julia v1.0 environment with i7 4800QM CPU and 16 GB RAM capacity. The SA parameters are used in the tests as: the maximum number of iterations is $10 \mathrm{~K}$, the maximum number of inner-iteration is 100 , the initial temperature level is 250 and, the temperature damping rate is 0.99 . The computational results are summarized in Table 2.

According to the results, the minimum total demand values are 9013.9 and 9906 as the simulated annealing algorithm with Julia and savings algorithm with Matlab, respectively. So, the objective function value is better with the proposed method as $9 \%$. On the other hand, as the savings algorithm is a constructive algorithm much better solution times were reached, however, for the numbers of required vehicles, the proposed approach requires at most 5 vehicles instead of 6 vehicles from savings algorithm. In addition, the more effective vehicle utilization rate is up to $88.72 \%$ from $86.27 \%$. Obtaining a better vehicle utilization rate may provide two important advantages. The first is the positive effect on the economy by reducing the fuel 
Table 1. Customer demands

\begin{tabular}{|c|c|c|c|c|c|c|c|c|c|c|c|c|}
\hline & P1 & P2 & P3 & P4 & P5 & P6 & P7 & P8 & P9 & P10 & P11 & P12 \\
\hline $\mathrm{C} 1$ & 225 & 275 & 585 & 300 & 575 & 625 & 325 & 650 & 425 & 550 & 405 & 475 \\
\hline $\mathrm{C} 2$ & 225 & 425 & 135 & 300 & 300 & 225 & 225 & 235 & 275 & 200 & 275 & 350 \\
\hline $\mathrm{C} 3$ & 450 & 225 & 230 & 235 & 600 & 775 & 375 & 270 & 500 & 200 & 575 & 150 \\
\hline C4 & 450 & 390 & 425 & 525 & 280 & 415 & 295 & 510 & 205 & 325 & 213 & 585 \\
\hline $\mathrm{C} 5$ & 530 & 200 & 225 & 265 & 285 & 350 & 200 & 225 & 425 & 650 & 250 & 370 \\
\hline C6 & 200 & 480 & 150 & 175 & 275 & 480 & 370 & 225 & 350 & 250 & 170 & 225 \\
\hline C7 & 375 & 725 & 375 & 510 & 575 & 200 & 570 & 550 & 380 & 275 & 450 & 375 \\
\hline C8 & 200 & 225 & 225 & 475 & 200 & 125 & 475 & 200 & 200 & 725 & 200 & 300 \\
\hline C9 & 525 & 625 & 665 & 650 & 270 & 615 & 200 & 475 & 395 & 300 & 630 & 380 \\
\hline $\mathrm{C} 10$ & 350 & 180 & 525 & 345 & 360 & 200 & 550 & 250 & 225 & 250 & 535 & 375 \\
\hline C11 & 375 & 385 & 550 & 525 & 450 & 530 & 400 & 440 & 200 & 450 & 605 & 200 \\
\hline C12 & 625 & 175 & 330 & 250 & 410 & 200 & 175 & 200 & 175 & 175 & 175 & 175 \\
\hline $\mathrm{C} 13$ & 555 & 250 & 775 & 450 & 525 & 525 & 420 & 525 & 370 & 695 & 675 & 430 \\
\hline C14 & 375 & 500 & 400 & 450 & 615 & 250 & 300 & 350 & 550 & 375 & 550 & 475 \\
\hline C15 & 375 & 625 & 750 & 385 & 450 & 475 & 400 & 675 & 725 & 900 & 300 & 275 \\
\hline C16 & 270 & 675 & 875 & 550 & 500 & 775 & 375 & 650 & 625 & 575 & 610 & 175 \\
\hline C17 & 700 & 375 & 530 & 475 & 275 & 325 & 650 & 725 & 515 & 550 & 300 & 175 \\
\hline C18 & 525 & 350 & 475 & 325 & 275 & 365 & 685 & 550 & 415 & 650 & 275 & 400 \\
\hline C19 & 175 & 500 & 250 & 500 & 375 & 225 & 735 & 350 & 375 & 575 & 475 & 625 \\
\hline $\mathrm{C} 20$ & 500 & 575 & 250 & 425 & 590 & 275 & 450 & 400 & 675 & 800 & 700 & 620 \\
\hline $\mathrm{C} 21$ & 475 & 300 & 570 & 525 & 525 & 300 & 680 & 175 & 650 & 725 & 450 & 325 \\
\hline $\mathrm{C} 22$ & 175 & 350 & 650 & 825 & 475 & 475 & 625 & 710 & 225 & 375 & 425 & 620 \\
\hline $\mathrm{C} 23$ & 450 & 315 & 400 & 300 & 250 & 775 & 775 & 435 & 400 & 415 & 510 & 545 \\
\hline $\mathrm{C} 24$ & 775 & 475 & 625 & 700 & 600 & 630 & 550 & 540 & 550 & 685 & 525 & 500 \\
\hline $\mathrm{C} 25$ & 750 & 575 & 800 & 625 & 750 & 500 & 475 & 675 & 750 & 300 & 525 & 525 \\
\hline $\mathrm{C} 26$ & 850 & 745 & 800 & 675 & 275 & 500 & 460 & 525 & 400 & 655 & 750 & 700 \\
\hline $\mathrm{C} 27$ & 485 & 215 & 375 & 675 & 650 & 505 & 585 & 590 & 600 & 625 & 750 & 425 \\
\hline $\mathrm{C} 28$ & 430 & 250 & 225 & 625 & 850 & 450 & 425 & 530 & 200 & 300 & 425 & 575 \\
\hline C29 & 250 & 625 & 675 & 600 & 580 & 420 & 375 & 255 & 350 & 200 & 250 & 650 \\
\hline $\mathrm{C} 30$ & 300 & 650 & 420 & 775 & 275 & 250 & 375 & 750 & 400 & 300 & 400 & 125 \\
\hline Total & 12945 & 12660 & 14265 & 14440 & 13415 & 12760 & 13500 & 13640 & 12530 & 14050 & 13378 & 12125 \\
\hline
\end{tabular}

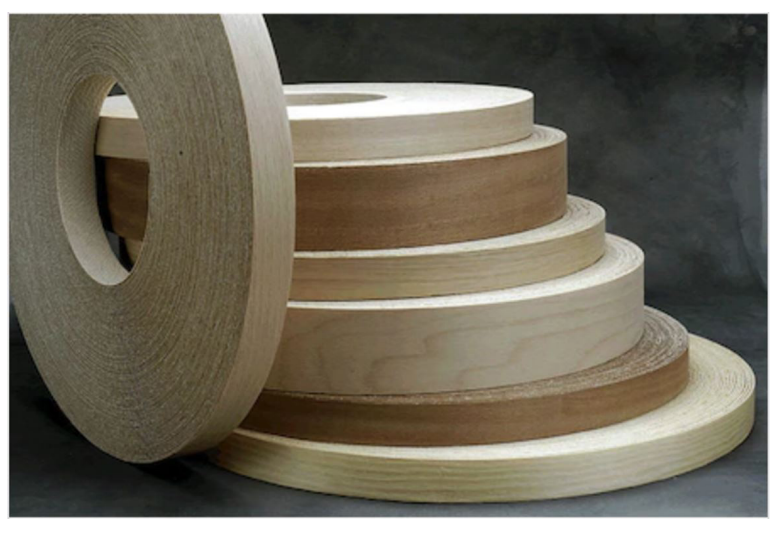

Figure 4. Furniture roving parts

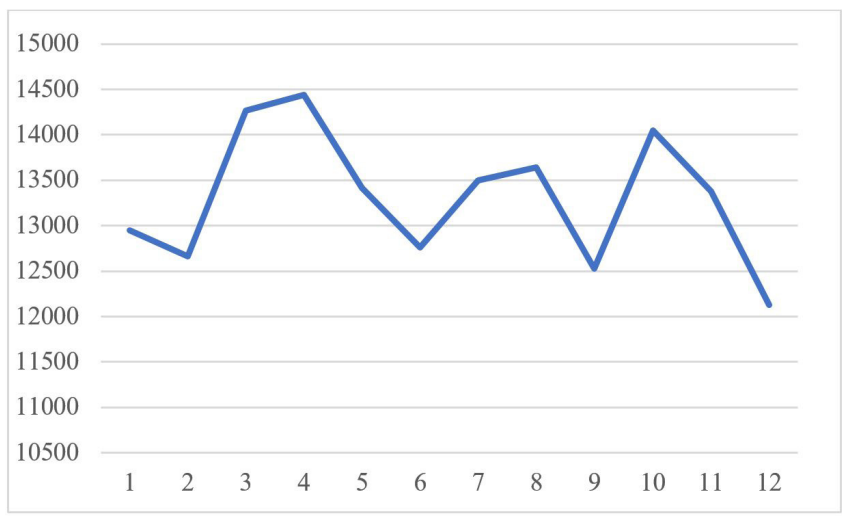

Figure 5. Total demand fluctuations for each period

consumption, and the second is the positive effect on the

environment by reducing carbon dioxide emissions. 
Table 2. Computational results

\begin{tabular}{|c|c|c|c|c|c|c|c|c|}
\hline & \multicolumn{9}{|c|}{ Simulated Annealing with Julia } & \multicolumn{3}{c|}{ Savings Algorithm with Matlab } \\
\hline & & & Sol Time & \# vehicle & \multicolumn{2}{|c|}{ Sol Time } & \# vehicle \\
\hline Dataset & Best & Worst & Avg & Avg & Number & Avg & Avg & Number \\
\hline P1 & 730 & 759 & 737.4 & 123.0 & 5 & 812 & 5 \\
\hline P2 & 733 & 764 & 744.6 & 128.3 & 5 & 834 & 5 \\
\hline P3 & 761 & 804 & 773.2 & 122.6 & 5 & 843 & 5 \\
\hline P4 & 763 & 910 & 799.2 & 120.0 & 5 & 840 & 5 \\
\hline P5 & 747 & 767 & 749.9 & 130.1 & 5 & 823 & 5 \\
\hline P6 & 735 & 755 & 739.7 & 127.7 & 5 & 824 & 5 \\
\hline P7 & 730 & 781 & 737.0 & 133.2 & 5 & 823 & 5 \\
\hline P8 & 752 & 811 & 764.5 & 125.0 & 5 & 837 & 5 \\
\hline P9 & 734 & 771 & 743.5 & 127.1 & 5 & 818 & 5 \\
\hline P10 & 744 & 753 & 746.1 & 128.6 & 5 & 820 & 5 \\
\hline P11 & 739 & 755 & 745.9 & 129.5 & 5 & 824 & 5 \\
\hline P12 & 726 & 737 & 732.9 & 131.2 & 5 & 808 & 5 \\
\hline
\end{tabular}

In this paper, a new scientific computing language Julia is used for solving CVRP with SA as the proposed approach. The CVRP model has investigated in a furniture roving part manufacturing company which is located in Denizli, Turkey. The company has the demand values from 30-customers for 12 periods. Before the scheduling implementation with Julia, they have no effective and efficient planning as they have been using spreadsheet programs for vehicle scheduling solutions. In this study, the solutions with Julia are used in practice for the distribution with higher utilization rate and minimum number of vehicles. The homogenous fleet is used with 3000 meters of vehicle capacity. The obtained results are compared to savings algorithms from Matlab programming language in terms of solution quality and time at the same problem.

In this study, the latest technology in the literature is taken into consideration in the developed SA code. However, it is not the SA algorithm that demonstrates the state-of-art. The authors aimed to achieve high-performance solutions by coding an industrial problem manually solved with a state-of-art language. Another state-of-art situation here is to solve a real vehicle routing problem using Julia language and SA and recommend it to the industry practitioners.

According to the results, the minimum total demand value for the simulated annealing algorithm is better than savings algorithm. The savings algorithm is a constructive algorithm. Therefore, solution times were better than simulated annealing algorithm. On the other hand, the simulated annealing algorithm requires at most 5 vehicles instead of 6 vehicles of the savings algorithm. This has a positive effect on the fuel economy and the environment.
Even if the savings algorithm has produced better solution time, the proposed approach has minimum total distance objective value, minimum number of required vehicles, and maximum vehicle utilization rate for the whole model. Furthermore, this study can be evaluated by starting to develop a new logistics toolbox with Julia scientific computing. So, as a further study, the VRP variants and the new and/or known solution methodologies with Julia can be considered.

The industrial problem handled in this study can be evaluated from different perspectives as a further study. For instance, the problem can be evaluated within the framework of inventory routing problems. Also, heterogeneous fleet vehicle routing problems, another complex vehicle routing problem, can be solved by these algorithms. Time window constraint can be added to deal with a more complex problem. The same industrial problem may arise as a more difficult vehicle routing problem in case of the state-of-art problems such as stochastic demand, green vehicle routing or electrical vehicle routing. When the industrial problem handled in this study and the theoretical approaches listed below are evaluated together, different expansions can be revealed for future studies.

\section{REFERENCES}

Abdulal, W., Jabas, A., Ramachandram, S. et al. (2012), "Task scheduling in grid environment using simulated annealing and genetic algorithm", in Maad, S. (Ed.), Grid Computing: Technology and Applications, Widespread Coverage and New Horizons, InTech, Croatia, pp. 89-110. 
Absi, N., Cattaruzza, D., Feillet, D. et al. (2017), "A relax-andrepair heuristic for the Swap-Body Vehicle Routing Problem", Annals of Operations Research, Vol. 253, No. 2, pp. 957-78.

Allahyari, S., Salari, M. and Vigo, D. (2015), “A hybrid metaheuristic algorithm for the multi-depot covering tour vehicle routing problem", European Journal of Operational Research, Vol. 242, No. 3, pp. 756-68.

Alvarenga, G.B., Mateus, G.R. and Tomi, G. (2007), “A genetic and set partitioning two-phase approach for the vehicle routing problem with time windows", Computers \& Operations Research, Vol. 34, pp. 1561-84.

Archetti, C., Feillet, D., Gendreau, M. et al. (2011), "Complexity of the VRP and SDVRP", Transportation Research Part C, Emerging Technologies, Vol. 19, No. 5, pp. 741-50.

Archetti, C., Fernández, E. and Huerta-Muñoz, D.L. (2017), "The flexible periodic vehicle routing problem", Computers \& Operations Research, Vol. 85, pp. 58-70.

Baños, R., Ortega, J., Gil, C. et al. (2013), “A simulated annealing-based parallel multi-objective approach to vehicle routing problems with time windows", Expert Systems with Applications, Vol. 40, No. 5, pp. 1696-707.

Barnhart, C. and Laporte, G. (Eds.). (2006), Transportation, Handbooks in Operations Research and Management Science, Vol. 14. North-Holland, Amsterdam.

Bektas, T., Demir, E. and Laporte, G. (2016) “Green vehicle routing", in Psaraftis, H.N. (Ed.), Green Transportation Logistics. Springer, Cham, pp. 243-65.

Bernal, J., Escobar, J.W., Paz, J.C. et al. (2018), “A probabilistic granular tabu search for the distance constrained capacitated vehicle routing problem", International Journal of Industrial and Systems Engineering, Vol. 29, No. 4, pp. 453-77.

Bertsimas, D.J., Chervi, P. and Peterson, M. (1995), "Computational approaches to stochastic vehicle routing problems", Transportation Science, Vol. 29, No. 4, pp. 342-52.

Bianchessi, N. and Irnich, S. (2019), "Branch-and-cut for the split delivery vehicle routing problem with time windows", Transportation Science, Vol. 53, No. 2, pp. 442-62.

Blum, C. and Roli, A. (2003), "Metaheuristics in combinatorial optimization: overview and conceptual comparison", ACM Computing Surveys, Vol. 35, No. 3, pp. 268-308.

Braysy, O. and Gendreau, M. (2005), "Vehicle routing problem with time windows, part I: route construction and local search algorithms", Transportation Science, Vol. 39, No. 1, pp. 104-18.

Brito, J., Moreno-Pérez, J.A. and Verdegay, J.L. (2009), "Fuzzy optimization in vehicle routing problems", in 2009 Conference of the International Fuzzy Systems Association and The European Society for Fuzzy Logic and Technology (IFSA) EUSFLAT), Lisbon, Portugal, pp. 1547-52.

Cacchiani, V., Hemmelmayr, V.C. and Tricoire, F. (2014), "A set-covering based heuristic algorithm for the periodic vehicle routing problem", Discrete Applied Mathematics, Vol. 163, pp. 53-64.

Cao, E. and Lai, M. (2010), "The open vehicle routing problem with fuzzy demands", Expert Systems with Applications, Vol. 37, No. 3, pp. 2405-11.

Chen, B., Rong, Q., Ruibin, B. et al. (2017), “An investigation on compound neighborhoods for VRPTW", in International Conference on Operations Research and Enterprise Systems, Springer, Cham, pp. 3-19.

Clarke, G. and Wright, J.W. (1964), "Scheduling of vehicles from a central depot to a number of delivery points", Operations Research, Vol. 12, pp. 568-81.

Coelho, V.N., Grasas, A., Ramalhinho, H. et al. (2016), “An ILS-based algorithm to solve a large-scale real heterogeneous fleet VRP with multi-trips and docking constraints", European Journal of Operational Research, Vol. 250, No. 2, pp. 367-76.

Cordeau, J.F., Gendreau, M., Hertz, A. et al. (2004), New Heuristics for the Vehicle Routing Problem, Technical Report G-2004-33, GERAD, Montreal, Canada.

Dantzig, G.B. and Ramser, J.M. (1959), "The truck dispatching problem", Management Science, Vol. 6, pp. 81-91.

Dorigo, M., Maniezzo, V. and Colorni, A. (1991), Positive Feedback as a Search Strategy, Technical Report N. 91-016, Politecnico di Milano, Italy.

Dror, M. (1994), "Note on the complexity of the shortest path models for column generation in VRPTW", Operations Research, Vol. 42, No. 5, pp. 977-8.

El Hassani, A.J., Bouhafs, L. and Koukam, A. (2008), "A hybrid ant colony system approach for the capacitated vehicle routing problem and the capacitated vehicle routing problem with time windows", in Caric, T. and Gold, H. (Eds.), Vehicle Routing Problem. I-Tech Education and Publishing KG, Vienna, Austria, pp. 59-70. 
Ene, S., Küçükoglu, I., Aksoy, A. et al. (2016), "A hybrid metaheuristic algorithm for the green vehicle routing problem with a heterogeneous fleet", International Journal of Vehicle Design, Vol. 71, No. 1-4, pp. 75-102.

Erdogan, S. and Miller-Hooks, E. (2012), "A green vehicle routing problem", Transportation Research Part E, Logistics and Transportation Review, Vol. 48, No. 1, pp. 100-14.

Farmer, J.D., Packard, N.H. and Perelson, A.S. (1986), "The immune system, adaptation, and machine learning", Physica, Vol. 22D, pp. 187-204.

Frazzon, E., Rodriguez, C.M., Pereira, M. et al. (2019), "Towards supply chain management 4.0", Brazilian Journal of Operations \& Production Management, Vol. 16, No. 2, pp. 180-91.

Gambardella, L.M., Taillard, É. and Agazzi, G. (1999), "MACS-VRPTW: a multiple colony system for vehicle routing problems with time windows", in Corne, D., Dorigo, M. and Glover, F. (Eds.), New Ideas in Optimization, McGraw-Hill, London, UK, pp. 63-76.

Ganesh, K. and Narendran, T.T. (2007), "CLOVES: a clusterand-search heuristic to solve the vehicle routing problem with delivery and pick-up", European Journal of Operational Research, Vol. 178, No. 3, pp. 699-717.

Gayialis, S.P., Konstantakopoulos, G.D. and Tatsiopoulos, I.P. (2019), "Vehicle routing problem for urban freight transportation: a review of the recent literature", in Sifaleras, A. and Petridis, K. (Eds.), Operational Research in the Digital Era: ICT Challenges, Springer Proceedings in Business and Economics, Springer, Cham, Sweden, pp. 89-104.

Gendreau, M., Jabali, O. and Rei, W. (2016), "50th anniversary invited article: future research directions in stochastic vehicle routing", Transportation Science, Vol. 50, No. 4, pp. 1163-73.

Glover, F. and McMillan, C. (1986), "The general employee scheduling problem: an integration of management science and artificial intelligence", Computers \& Operations Research, Vol. 13, No. 5, pp. 563-93.

Gribkovskaia, I., Laporte, G. and Shyshou, A. (2008), "The single vehicle routing problem with deliveries and selective pickups", Computers \& Operations Research, Vol. 35, No. 9, pp. 2908-24.

Hartl, R.F., Hasle, G. and Janssens, G.K. (2006), "Special issue on rich vehicle routing problems", Central European Journal of Operations Research, Vol. 14, No. 2, pp. 103-4.
Holland, J.H. (1975), Adaptation in Natural And Artificial Systems, University of Michigan Press, Ann Arbor, MI.

Huber, S. and Geiger, M.J. (2017), “Order matters: a variable neighborhood search for the swap-body vehicle routing problem", European Journal of Operational Research, Vol. 263, No. 2, pp. 419-45.

Johnson, D.S., Aragon, C.R., McGeoch, L.A. et al. (1989), "Optimization by simulated annealing: an experimental evaluation; part I, graph partitioning", Operations Research, Vol. 37, No. 6, pp. 865-92.

Julia Language (2018), available at: www.julialang.org (accessed 15 December 2018).

Kara, I. and Derya, T. (2011), "Polynomial size formulations for the distance and capacity constrained vehicle routing problem", in AIP Conference Proceedings, AIP, Maryland, pp. 1713-8.

Karaboga, D. (2005), An Idea Based on Honey Bee Swarm for Numerical Optimization, TR-06, Erciyes University, Engineering Faculty, Computer Engineering Faculty, Kayseri, Turkey.

Karagul, K., Sahin, Y., Aydemir, E. et al. (2019), “A simulated annealing algorithm based solution method for a green vehicle routing problem with fuel consumption", in Lean and Green Supply Chain Management, Springer, Cham, pp. 161-87.

Karagul, K., Tokat, S. and Aydemir, E. (2016), "A new algorithm to the construction of the initial routes for the capacitated vehicle routing problem", Journal of Engineering Sciences and Design, Vol. 4, No. 3, pp. 215-26.

Katoh, N. and Yano, T. (2006), "An approximation algorithm for the pickup and delivery vehicle routing problem on trees", Discrete Applied Mathematics, Vol. 154, No. 16, pp. 2335-49.

Kennedy, J. and Eberhart, C. (1995), "Particle swarm optimization", in IEEE International Conference on Neural Networks, Piscataway, NJ, pp. 1942-8

Kirkpatrick, S., Gelatt, C.D. and Vecchi, M.P. (1983), "Optimization by simulated annealing", Science, Vol. 220, No. 4598 , pp. 671-80.

Koç, Ç. and Karaoglan, I. (2016), "The green vehicle routing problem: a heuristic based exact solution approach", Applied Soft Computing, Vol. 39, pp. 154-64.

Koç, Ç., Erbaş, M. and Ozceylan, E. (2018), "A rich vehicle routing problem arising in the replenishment of automated 
teller machines", An International Journal of Optimization and Control: Theories \& Applications, Vol. 8, No. 2, pp. 276-87.

Koç, Ç., Erbaş, M. and Özceylan, E. (2019) "The impact of routing on $\mathrm{CO} 2$ emissions at a retail grocery store chain: a GIS-based solution approach", in Paksoy, T., Weber, G.W. and Huber, S. (Eds.), Lean and Green Supply Chain Management, International Series in Operations Research \& Management Science, Vol. 273, Springer, Cham, Sweden, pp. 143-60.

Kuo, R.J., Wibowo, B.S. and Zulvia, F.E. (2016), “Application of a fuzzy ant colony system to solve the dynamic vehicle routing problem with uncertain service time", Applied Mathematical Modelling, Vol. 40, No. 23-24, pp. 9990-10001.

Lalla-Ruiz, E. and Voß, S. (2019), "A popmusic approach for the multi-depot cumulative capacitated vehicle routing problem", Optimization Letters, Vol. 19, pp. 1-21.

Laporte, G. (1992), "The vehicle routing problem: an overview of exact and approximate algorithms", European Journal of Operational Research, Vol. 59, pp. 345-58.

Laporte, G. (2009), "Fifty years of vehicle routing", Transportation Science, Vol. 43, No. 4, pp. 408-16.

Laporte, G. and Semet, F. (2002). "Classical heuristics for the capacitated VRP", in Toth, P. and Vigo, D. (Eds.), The Vehicle Routing Problem, SIAM Monographs on Discrete Mathematics and Applications, SIAM, Philadelphia, pp. 109-28.

Laporte, G., Louveaux, F. and Mercure, H. (1992), "The vehicle routing problem with stochastic travel times", Transportation Science, Vol. 26, No. 3, pp. 161-70.

Laporte, G., Mercure, H. and Nobert, Y. (1986), "An exact algorithm for the asymmetrical capacitated vehicle routing problem", Networks, Vol. 16, No. 1, pp. 33-46.

Laporte, G., Nobert, Y. and Taillefer, S. (1988), "Solving a family of multi-depot vehicle routing and location-routing problems", Transportation Science, Vol. 22, No. 3, pp. 161-72.

Letchford, A.N., Lysgaard, J. and Eglese, R.W. (2007), "A branch-and-cut algorithm for the capacitated open vehicle routing problem", The Journal of the Operational Research Society, Vol. 58, No. 12, pp. 1642-51.

Leung, S.C.H., Zheng, J., Zhang, D. et al. (2010), "Simulated annealing for the vehicle routing problem with twodimensional loading constraints", Flexible Services and Manufacturing Journal, Vol. 22, No. 1, pp. 61-82.
Li, B., Guo, C., Ning, T. et al. (2017), "Solve VRPPD with improved bacteria optimization algorithm", in 2017 2nd International Conference on Materials Science, Machinery and Energy Engineering (MSMEE 2017), Dalian, China, Atlantis Press.

Lin, L. and Fei, C. (2012), "The simulated annealing algorithm implemented by the MATLAB", International Journal of Computer Science Issues, Vol. 9, No. 6, pp. 357-60.

Mazzeo, S. and Loiseau, I. (2004), "An ant colony algorithm for the capacitated vehicle routing", Electronic Notes in Discrete Mathematics, Vol. 18, pp. 181-6.

Montoya, A., Guéret, C., Mendoza, J.E. et al. (2016), "A multi-space sampling heuristic for the green vehicle routing problem", Transportation Research Part C, Emerging Technologies, Vol. 70, pp. 113-28.

Mosheiova, G. (1998), "Vehicle routing with pick-up and delivery: tour-partitioning heuristics", Computers \& Industrial Engineering, Vol. 34, No. 3, pp. 669-84.

Osman, I.H. (1993), "Metastrategy simulated annealing and tabu search algorithms for the vehicle routing problem", Annals of Operations Research, Vol. 41, pp. 421-51.

Penna, P.H.V., Subramanian, A., Ochi, L.S. et al. (2019), “A hybrid heuristic for a broad class of vehicle routing problems with heterogeneous fleet", Annals of Operations Research, Vol. 273, No. 1-2, pp. 5-74.

Pichpibul, T. and Kawtummachai, R. (2013), "A heuristic approach based on clarke-wright algorithm for open vehicle routing problem", The Scientific World Journal, Vol. 2013, pp. 1-11.

Rahimi-Vahed, A., Crainic, T.G., Gendreau, M. et al. (2015), "Fleet-sizing for multi-depot and periodic vehicle routing problems using a modular heuristic algorithm", Computers \& Operations Research, Vol. 53, pp. 9-23.

Reil, S., Bortfeldt, A. and Mönch, L. (2018), "Heuristics for vehicle routing problems with backhauls, time windows, and 3D loading constraints", European Journal of Operational Research, Vol. 266, No. 3, pp. 877-94.

Şahin, Y. and Eroğlu, A. (2014), "Metaheuristic methods for capacitated vehicle routing problem: literature review", Suleyman Demirel University the Journal of Faculty of Economics and Administrative Sciences, Vol. 19, No. 4, pp. 337-55. 
Saint-Guillain, M., Solnon, C. and Deville, Y. (2017), "The static and stochastic VRP with time windows and both random customers and reveal times", in European Conference on the Applications of Evolutionary Computation, Springer, Cham, pp. 110-27.

Salhi, S., Imran, A. and Wassan, N.A. (2014), "The multidepot vehicle routing problem with heterogeneous vehicle fleet: formulation and a variable neighborhood search implementation", Computers \& Operations Research, Vol. 52, pp. 315-25.

Silva Júnior, O., Lopes, L.A. and Bergmann, U. (2011), “A free geographic information system as a tool for multi-depot vehicle routing", Brazilian Journal of Operations \& Production Management, Vol. 8, No. 1, pp. 103-20.

Sim, K., Hart, E., Urquhart, N. et al. (2019), "A new rich vehicle routing problem model and benchmark resource", in Minisci, E., Vasile, M., Periaux, J. et al. (Eds.), Advances in Evolutionary and Deterministic Methods for Design, Optimization and Control in Engineering and Sciences, Springer, Cham, pp. 503-18.

Taillard, É.D. (1993), "Parallel iterative search methods for vehicle routing problems", Networks, Vol. 23, pp. 661-76.

Tarantilis, C.D., Ioannou, G., Kiranoudis, C.T. et al. (2004), "A threshold accepting approach to the open vehicle routing problem", Operations Research, Vol. 38, No. 4, pp. 345-60.

Tavakkoli-Moghaddam, R., Gazanfari, M., Alinaghian, M. et al. (2011), "A new mathematical model for a competitive vehicle routing problem with time windows solved by simulated annealing", Journal of Manufacturing Systems, Vol. 30, No. 2, pp. 83-92.

Tavakkoli-Moghaddam, R., Safaei, N., Kah, M.M.O. et al. (2007), "A new capacitated vehicle routing problem with split service for minimizing fleet cost by simulated annealing", Journal of the Franklin Institute, Vol. 344, No. 5, pp. 406-25.

Toth, P. and Vigo, D. (2002), "VRP with backhauls", in Toth, P. and Vigo, D., The Vehicle Routing Problem. Society for Industrial and Applied Mathematics, Philadelphia, pp. 195-224.

Tyasnurita, R., Özcan, E. and John, R. (2017), “Learning heuristic selection using a time delay neural network for open vehicle routing", in 2017 IEEE Congress on Evolutionary Computation (CEC), IEEE, San Sebastian, pp. 1474-81.

Vincent, F.Y., Redi, A.P., Hidayat, Y.A. et al. (2017), “A simulated annealing heuristic for the hybrid vehicle routing problem", Applied Soft Computing, Vol. 53, pp. 119-32.

Wu, T.-H., Low, C. and Bai, J.-W. (2002), "Heuristic solutions to multi-depot location-routing problems", Computers \& Operations Research, Vol. 29, No. 10, pp. 1393-415.

Yanik, S. and Bozkaya, B. (2014), “A new VRPPD model and a hybrid heuristic solution approach for e-tailing", European Journal of Operational Research, Vol. 236, No. 3, pp. 879-90.

Zeng, L., Ong, H.L. and Ng, K.M. (2005), “An assignmentbased local search method for solving vehicle routing problems", Asia-Pacific Journal of Operational Research, Vol. 22, pp. 85-104.

Received: 06 Jun 2019

Approved: 17 Nov 2019

How to cite: Aydemir, E. and Karagul, K. (2020), "Solving a periodic capacitated vehicle routing problem using simulated annealing algorithm for a manufacturing company", Brazilian Journal of Operations \& Production Management, Vol. 17, No. 1, e2020866. https://doi.org/10.14488/BJOPM.2020.011 


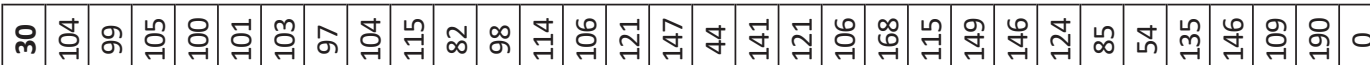
ని 숙

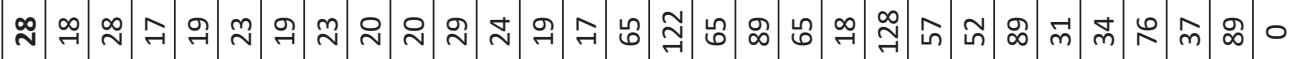
ヘ

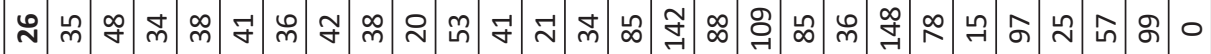

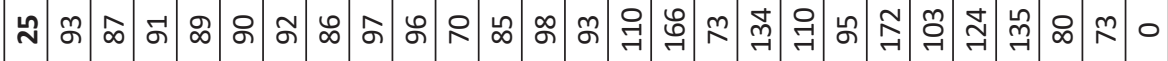

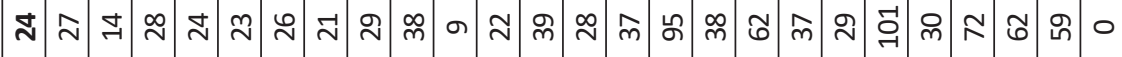
ఇ $\tilde{m}$ ட

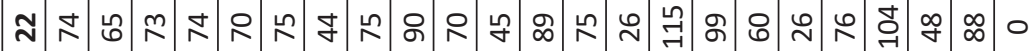

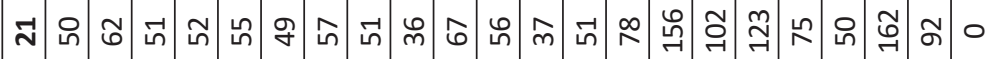

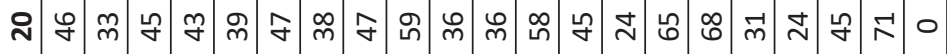

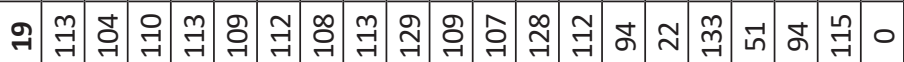

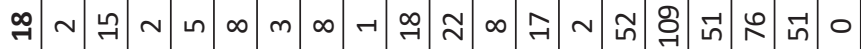

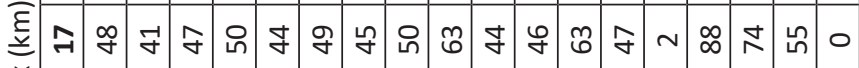

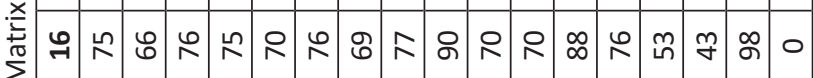

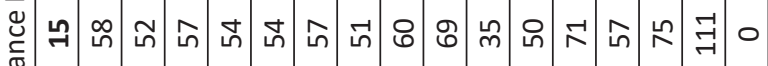

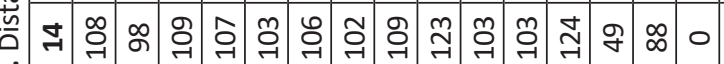

爻

$\frac{0}{\frac{0}{2}}$

ت

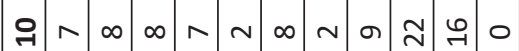

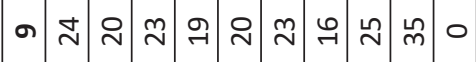

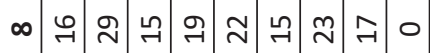

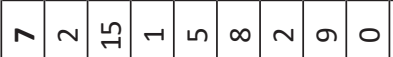

$\begin{array}{lllllllll}0 & \wedge & \infty & \infty & \wedge & n & \sim & 0\end{array}$

$\begin{array}{lllllllll}n & -1 & \infty & -1 & m & m & 0\end{array}$

$\infty \infty_{0} 0$

$\begin{array}{rllllll}0 & 0 & \text { in } & 0 & 0\end{array}$

m $m$ I $m$

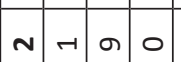

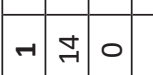

0 O

○ 